\title{
Hitting the brakes on ectopic lymphoid structure formation
}

A subset of patients with rheumatoid arthritis (RA) develop ectopic lymphoid-like structures (ELSs) in inflamed joints. These lymphoid aggregates contribute to the differentiation and activation of T cells and B cells, and are associated with disease severity and autoantibody production in RA. In a new study published in The Journal of Experimental Medicine, Gareth Jones and colleagues analysed experimental models of arthritis and ultrasound-guided synovial biopsies from patients with RA and identified IL-27 as a negative regulator of ELS formation.

"Our study focused on a particular subtype of RA pathology, the follicular pathotype, which is associated with severe symptoms and poor response to some of the mainstream therapies," Jones describes. In these patients, ELSs develop in the inflamed joint tissue and contribute to local pathology.

Previous research showed that IL-27, a cytokine that inhibits the expansion of $\mathrm{CD}^{+} \mathrm{T}$ helper $\left(\mathrm{T}_{\mathrm{H}}\right) 17$ cells, could ameliorate joint pathology and prevent osteoclastogenesis, suggesting that IL-27 could have an important role in synovial histopathology. By using an antigeninduced arthritis model in mice deficient for Il27ra (the gene encoding the IL-27 receptor a chain), the researchers observed more severe disease in mice lacking Il27ra than in wild-type controls. These observations included evidence of increased leukocyte infiltration, hypertrophy, synovial exudates and cartilage and bone tissue degradation; $\mathrm{Il}_{2} \mathrm{7 \textrm {ra } ^ { - / - }}$ mice also showed signs of enhanced activation of adaptive immunity, with increased antigen-specific IgG titres, higher numbers of $\mathrm{T}_{\mathrm{H}} 17$ cells in draining lymph nodes and higher serum levels of IL-17 than wild-type mice. Notably, the signs of enhanced pathology observed in $\mathrm{Il}_{2} 7 \mathrm{ra}^{-/-}$mice were associated with larger $\mathrm{CD}^{+}$cellular aggregates in the inflamed synovium at day 10 of the disease course $\left(12.8 \times 10^{4} \pm 1.7 \mu \mathrm{m}^{2}\right.$ versus $1.6 \times 10^{4} \pm 0.5 \mu \mathrm{m}^{2}$ in wild-type mice). The lymphoid aggregates found in $\mathrm{Il}_{2} 7 \mathrm{ra}^{-/}$ mice resembled ELSs and expressed $B c l 6$, a marker of $\mathrm{T}$ follicular helper $\left(\mathrm{T}_{\mathrm{FH}}\right)$ cells and germinal centre B cells, and CD21, a marker of follicular dendritic cells.

The inflamed synovia of $127 \mathrm{ra}^{-/-}$mice also expressed higher levels of $\mathrm{Cxcl} 13$ and Ccl21, which encode chemokines involved in the spatial organization of ELSs, on day 3 after disease induction. On day 10 , the expression of Lta and $L t b$ (the genes encoding the lymphoneogenic cytokines lymphotoxin $\alpha$ and lymphotoxin $\beta$ ) and $\operatorname{Prdm} 1$ (which encodes PR domain zinc finger protein 1, or Blimp1, a B-cell differentiation and plasma cell factor) were also increased in $\mathrm{Il} 27 \mathrm{ra}^{-/-}$mice compared with wild-type controls. In agreement with the known inhibitory effect of IL-27 on $\mathrm{T}_{\mathrm{H}} 17$ responses, $127 \mathrm{ra}^{-/-}$mice expressed higher levels of IL-17 in the inflamed synovium.

Next, Jones et al. sought to confirm these findings in patients with RA. The researchers classified synovial samples from patients with RA according to the presence or absence of ELSs. Compared with ELS- biopsies, and similarly to the data from the experimental arthritis model, ELS ${ }^{+}$samples had increased expression of IL17 and IL21 and, importantly, expressed lower levels of IL27. Additionally, levels of synovitis and numbers of infiltrating $\mathrm{CD}^{+}$and $\mathrm{CD} 20^{+}$ cells were inversely correlated with $I L 27$ expression levels. These results led the authors to propose that IL-27 levels in patients with RA could be predictive of diffuse synovial histopathology. Furthermore, Jones anticipates that "the detection of IL-27 in the joints may be used as a predictive marker for diagnostic purposes and decision-making regarding the best course of therapy for patients with ELS-associated diseases."

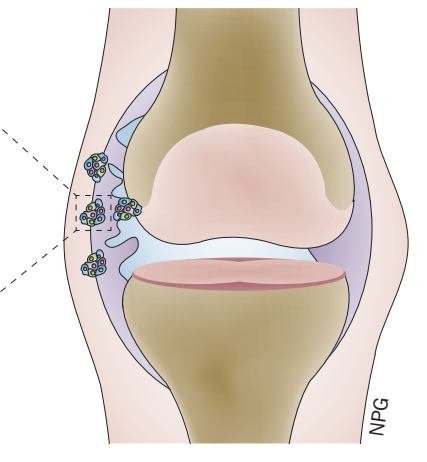

Dominique Baeten of the Academic Medical Center, University of Amsterdam, who was not involved in this study, cautions that "the presence of synovial ELSs is not a fixed feature, and these can disappear and reappear over time in a single patient." As such, whether the presence of ELSs can be considered a determinant phenotype in patients with RA remains unclear. Baeten concludes that despite the increase in ELSs observed in $\mathrm{Il} 27 \mathrm{ra}^{-/-}$mice, "there is no demonstration of a causal relationship, nor of the direction of the causal relationship. What is really missing in the current study is the direct demonstration that increasing IL-27 signalling decreases ELSs."

Despite these open questions, the fact that patients with $\mathrm{ELS}^{+}$synovitis have been reported to have weak responses to anti-TNF therapies lends special relevance to these findings. "This is the first study describing IL-27 as a regulator of ELS development in chronic inflammatory diseases," states Jones. "Our data, together with previously described anti-inflammatory roles for IL-27 in chronic inflammatory conditions, highlights the therapeutic potential of this cytokine."

The mechanisms that regulate IL27 expression in the context of joint inflammation remain unknown, but this cytokine could become a target of future therapies owing to its role in the formation of ELSs, and ultimately on the production of pathogenetic autoantibodies and on the destruction of cartilage and bone tissue.

João H. Duarte

Original article Jones, G. W. et al. Interleukin-27 inhibits ectopic lymphoid-like structure development in early inflammatory arthritis. J. Exp. Med. doi:10.1084/ jem.20132307 